\title{
Valoración de los factores que determinan la atracción laboral de las empresas desde la perspectiva de los futuros colaboradores
}

\author{
Ivette M. Durán-Seguel(1), Maximiliano E. Gallegos(2), Gonzalo A. Dauvin ${ }^{(1)}$ y Javiera A. Rojas ${ }^{(1)}$ \\ (1) Facultad de Ciencias Sociales y Económicas, Departamento de Economía y Administración, Universidad Católica del \\ Maule, Avda. San Miguel 3605, Talca, Chile. (correo-e: iduran@ucm.cl; gonzalodauvin@gmail.com; \\ javirojasg22@gmail.com) \\ (2) Facultad de Economía y Negocios, Universidad de Talca, Talca, Chile. (correo-e: mgallegos@utalca.cl)
}

Recibido Ene. 7, 2020; Aceptado Mar. 11, 2020; Versión final May. 12, 2020, Publicado Oct. 2020

\section{Resumen}

Este estudio analiza los factores de atracción y retención laboral más valorados por alumnos de último año y recién egresados de las tres principales facultades de negocio de la región del Maule (Chile). Esta investigación tiene enfoque cuantitativo no experimental y diseño de carácter descriptivo. Para medir se utilizó la escala de atractivo del empleador. Los principales resultados indican que, 1) los futuros colaboradores valoran el conocimiento de marca del empleador; 2) existen diferencias en la valoración de los factores por género, mientras las mujeres relevan el crecimiento, desarrollo y reconocimiento, los hombres valoran más un ambiente de trabajo innovador, dinámico y competitivo; y 3) quienes aún no egresan, aprecian más los trabajos innovadores, creativos y responsables socialmente. Las conclusiones apuntan a actualizar los procesos de atracción y retención de talentos, considerando en sus estrategias las necesidades de las nuevas generaciones y las diferencias del atractivo del empleador por género.

Palabras clave: marca del empleador; empleo juvenil; recursos humanos; universidades y atracción laboral

\section{Assessment of the factors that determine job appeal in companies from the perspective of future employees}

\begin{abstract}
This research study analyses the most valued job attraction and retention factors of senior students and recent graduates from three business schools in the Maule region (Chile). This research has a non-experimental quantitative approach and a descriptive design. The employer attractiveness scale was used to quantify the different factors. The results indicate that future collaborators value the knowledge of the employer brand. There are also differences in the assessment of factors by gender. Women give more importance to growth, development, and recognition while men value a more innovative, dynamic, and competitive work environment. In addition, senior students appreciate a more innovative, creative, and socially responsible work environment. In conclusion, talent attraction and retention processes are updated by considering within their strategies the needs of new generations and the differences of employer attraction by gender.
\end{abstract}




\section{INTRODUCCIÓN}

En las últimas décadas las organizaciones han relevado la necesidad de desarrollar estrategias que les permitan atraer y retener personas talentosas. En este sentido, la imagen que proyectan, tanto hacia sus actuales como futuros colaboradores, se ha transformado en un elemento clave para alcanzar este objetivo (Eger, et al., 2019). La marca del empleador (EB por sus siglas en inglés), también conocida como employer branding, está compuesta por beneficios tangibles e intangibles, es una expresión de la cultura, valores, comportamiento y prácticas de negocio de las empresas, que se conjugan para proyectar una imagen, la que a su vez afecta el sentido de pertenencia de los colaboradores e impacta la atracción de nuevos empleados. El vínculo entre empresa y trabajadores se fortalece, al lograr un posicionamiento de imagen entre los grupos de interés o stakeholders de la compañía (Berthon et al., 2005; Bussin y Mouton, 2019). Para las organizaciones es importante garantizar que sus colaboradores se involucren en su cultura y estrategia organizacional, para lo cual están utilizando el EB (Eger, et al., 2019). Sin embargo, la marca del empleador es aún un concepto en desarrollo (Liu, 2018).

En relación a la interrogante de ¿qué buscan los jóvenes en el trabajo?, de acuerdo con Kucherov, et al., (2019), ellos buscan una cultura empresarial atractiva en términos de diversidad, equilibrio entre vida personal y trabajo, son amantes de los retos por lo cual están dispuestos a asumir responsabilidades, buscan tener autonomía y flexibilidad para trabajar desde un tercer lugar, y liderazgos con impactos positivos. En este segmento, quiénes tienen educación superior universitaria, tienen además como reto lograr una mayor capacidad de adaptación de sus competencias a las demandas del mercado laboral, para tener mayor posibilidad de empleo (Albert-Gómez, et al., 2017). De esta forma, el gran desafío para las empresas es plantearse cómo atraer a jóvenes talentos al proceso de reclutamiento, además, de organizar el trabajo, idear nuevas prácticas laborales que resulten atractivas para los nuevos jóvenes profesionales, y proyectar desde sus organizaciones liderazgos con impactos positivos en la sociedad (Eger, et al., 2019; Brymer, et al., 2014; Kucherov, et al., 2019). Por otra parte, Ramoni y Orlandoni (2016) expone que, a pesar del creciente nivel educativo de los jóvenes, las condiciones laborales difieren según el género, donde los resultados soportan la tesis de mercado laboral segmentado, con claras diferencias tanto en salarios como en sectores y condiciones de empleo y, además, con un cierto rezago en la evolución del mercado laboral de las mujeres. En relación a los salarios, si bien hay autores que reconocen la importancia de otros factores por sobre el económico, no hay total consenso al respecto, específicamente en relación al grupo objetivo (Mičík y Mičudová, 2018).

Estudios realizados en diferentes partes del mundo, apuntan a que las áreas de recursos humanos debiesen considerar la opinión de los futuros colaboradores, es necesario que tanto las campañas de EB y las estrategias de reclutamiento consideren las diferencias en la importancia de los factores, entre los grupos objetivo de diferentes géneros o culturas (Eger, et al., 2019). Por otra parte, las necesidades de las organizaciones han ido cambiando y actualmente las condiciones de la economía digital demandan nuevas competencias que se exigen a las empresas y para las cuales requieren trabajadores que las adquieran más rápidamente, y en este contexto son los jóvenes quienes tienen una mayor ventaja (Kucherov, et al., 2019). Además, son ellos los principales embajadores de la organización en sus aspectos positivos como negativos, según, Veas-González, et al., (2019), mientras más satisfechos esté el grupo objetivo existe una mayor propensión a quedarse en la organización. En Chile los jóvenes representan el 37\% de la fuerza laboral (Deloitte Chile, 2018). Este grupo es bastante particular en cuanto a sus necesidades y aspiraciones, la mayoría de esta generación poseen estudios técnicos o profesionales, su poder adquisitivo es superior que el de sus padres, están totalmente conectados a través de las redes sociales y han vivido el acelerado cambio tecnológico de las últimas décadas (Kucherov, et al., 2019). Esta generación de jóvenes es denominada millennials, son definidos por Kucherov, et al., (2019), como "independientes, socialmente activos, tienen un alto nivel de autosuficiencia y les gusta trabajar en equipos" (p.32). Además, asignan gran importancia a la ética, tanto en la elección como en la lealtad al lugar de trabajo donde se desarrollan (Kucherov, et al., 2019; Mičík y Mičudová, 2018).

Este artículo se centra en los jóvenes que estudian carreras en el área de negocios, en universidades de la región del Maule. Esta zona, se caracteriza por ser la región con la menor proporción de trabajadores profesionales, científicos e intelectuales del país, con un 11,9\% del total de ocupados INE (2018). En cuanto a la tasa de participación en la fuerza laboral según sexo, los hombres muestran una participación de 61,7\% y las mujeres un $38,2 \%$ INE (2018). De acuerdo con las estadísticas de empleabilidad aquí planteadas, existiría un escenario adverso para los jóvenes profesionales en la región del Maule, especialmente para las mujeres. Esto, sumado a la falta de experiencia de los recién egresados, explicaría, en parte, porque los nuevos profesionales enfocan su búsqueda de empleo en otras ciudades de mayor tamaño, principalmente en la capital del país (Santiago de Chile). En este sentido, parece relevante conocer cuáles son los factores que los jóvenes a punto de egresar de las universidades o recién egresados valoran más en relación a la marca del empleador y que pueden hacer la diferencia al momento de decidir si se quedan en la región o no. 
En consecuencia, el objetivo de esta investigación es conocer cuáles son los factores de atracción y retención laboral, más valorados por los alumnos de últimos años y recién egresados de las tres principales escuelas de negocio de la región del Maule. De esta forma, se busca entregar información para la toma de decisiones estratégicas, en el ámbito de recursos humanos, para empresas de la región del Maule, que les permita mejorar su posicionamiento en el mercado laboral.

\section{OTROS ANTECEDENTES}

Las organizaciones que aspiran tener éxito, reconocen que es necesario desarrollar ventajas competitivas, y en el último tiempo se ha reconocido y valorado al recurso humano como un factor estratégico y motor de dichas ventajas (Babikova y Bucek, 2019). Asimismo, para Deepa y Baral, (2017), las empresas deben dar prioridad a la atracción y retención del talento humano, para así beneficiarse de contar con colaboradores capacitados, que generen valor para la empresa. Quienes dirigen las organizaciones, deben estar conscientes de que solo pueden crecer, prosperar y mantener su continuidad, si son capaces de optimizar el beneficio percibido por todos sus grupos de interés, especialmente el de sus trabajadores. La necesidad de contar con los mejores colaboradores (talentos) radica en el impacto directo que tienen sobre todas las actividades de la organización, y por tanto son claves para lograr ventajas competitivas (Deepa y Baral, 2017).

Luego, uno de los mayores retos que enfrentan las organizaciones es atraer y retener trabajadores calificados, que cuenten con las competencias necesarias para hacer frente a las diferentes situaciones externas que afectan a las empresas, por lo que retener talento es de suma importancia para el crecimiento de la organización, porque marcas positivas generan organizaciones atractivas (Eger, et al., 2019), tanto para retener como para atraer. En este sentido, como una manera de enfrentar este reto y asegurar que el personal calificado se quede en la empresa, comienza a desarrollarse el concepto de marketing interno, el cual mezcla los conceptos de mercadotecnia tradicional, pero apuntándolos hacia el interior de la organización, considerando a los empleados como clientes internos, reconociendo su esfuerzo y dedicación, tanto físico como mental, todo ello con el fin de promocionar la imagen de marca de la empresa entre los colaboradores y fomentar así la identidad corporativa, (De Bruin-Reynolds, et al., 2015).

Posteriormente, estás técnicas de marketing comenzaron a ser orientadas también hacia el mercado laboral, con la finalidad de atraer nuevos colaboradores que se sintiesen atraídos por valores e identidad de la organización, llegando a convertirse en una confluencia de diferentes ámbitos de la administración, como son los recursos humanos, marketing y comportamiento organizacional, además, de la psicología (Babikova y Bucek, 2019), esta intersección es lo que hoy conocemos como la marca del empleador (EB). En consecuencia, construir una marca de empleador parece ser una de las soluciones a largo plazo para convertirse en un empleador de elección y así, atraer y retener al mejor talento, (Deepa y Baral, 2017).

\section{Marca del empleador (EB)}

La marca del empleador (EB), es un término acuñado por primera vez por Ambler y Barrow (1996), quiénes la definieron como: "un paquete de beneficios funcionales, económicos y psicológicos proporcionados por el empleo e identificados con la empresa empleadora" (p.187). Desde esta definición han existido varias de las cuales se considerará la expresada por Backhaus (2016), como la "identidad de marca que caracteriza al empleador" (p.196), empatizando con el sentido neutral del concepto original. Otros autores han dejado de lado la neutralidad y sus definiciones se han reducido, enfocándose en ser el empleador elegido (Backhaus, 2016). Otros, indican que el objetivo principal del EB es presentar una imagen positiva y atractiva tanto para las personas que trabajan actualmente en la organización como para quienes buscan empleo (Kucherov, et al., 2019).

La identidad de marca del empleador, implica un conjunto de actividades que realiza la organización para construir y promover la marca (Backhaus y Tikoo, 2004), donde la diferenciación del empleador en el mercado laboral, proviene de la atracción de talentos y la mantención del compromiso de los empleados con la organización. En otras palabras, la aspiración de las organizaciones a través de la gestión de la marca del empleador, busca fortalecer la imagen de marca de la empresa tanto entre los actuales como futuros colaboradores de la organización (Deepa y Baral, 2017). Las empresas son cada vez más conscientes de que todas sus actividades, están sujetas a la percepción positiva o negativa, tanto por parte de sus colaboradores como de la comunidad en su conjunto, y a su vez, la imagen que éstas proyectan afecta el nivel de compromiso de sus colaboradores y la atracción de nuevos empleados talentosos (Deepa y Baral, 2017). Una imagen de marca del empleador afecta positiva o negativamente, el atractivo de la organización para los solicitantes de empleo, no es neutra, por lo cual se debe potenciar como una fortaleza y no como una debilidad (Kucherov, et al., 2019). Las ideas o sensaciones que la imagen de marca del empleador genera, pueden estar asociadas al producto o servicio ofrecido por la organización, como a los beneficios que ésta brinda, y por lo general solo se relaciona con sus productos y no en la proyección sobre sus trabajadores (Maxwell y Knox, 2009; Miles y Mangold, 2004). 
La conexión con el colaborador se da cuando en la imagen que proyecta el empleador muestra todos los valores, características y beneficios que puede obtener un trabajador al unirse a la organización, y que son de interés para él, por lo que la imagen del empleador afecta a un solicitante de empleo y provoca atracción hacia la empresa en base a la concordancia que haya con sus valores y características personales (Berthon, et al., 2005). Backhaus y Tikoo (2004), insisten en la importancia del mensaje que el empleador proyecte en cuanto a valores, beneficios y contribuciones, éste debe ser objetivo y real para que no haya una decepción por parte del trabajador y la marca del empleador sea efectiva. En relación a la lealtad de los colaboradores a la marca del empleador, surge como consecuencia de la fuerte cultura e identidad de la organización (Kucherov, et al., 2019). Una experiencia positiva con la marca empleadora es importante para que el trabajador desarrolle un compromiso afectivo hacia ésta, lo cual provoca una relación de apego con la institución y los objetivos que se desean alcanzar, alentando la motivación del personal (Eger, et al.,2019). De esta forma, se alimentan las percepciones de la identidad de la organización y su cultura lo que conduce a la lealtad (deslealtad) con la organización (Backhaus, 2016). En esta misma dirección, Love y Singh (2011) afirman que la cultura genera un modelo de conducta y disposición para colaboradores frente a labores diarias. Otro componente actitudinal hacia a la lealtad de la marca, es la identidad o sentido de pertenencia, de las personas que trabajan en la organización. En este aspecto, Kucherov, et al., (2019), identificó en su investigación que los jóvenes profesionales de negocios esperan mayores inversiones en su crecimiento personal y profesional, y retribuyen con lealtad y compromiso con el empleador.

Por otra parte, se postula que tanto la identidad organizacional percibida (percepción interna), así como la imagen externa interpretada (percepción interna sobre qué piensan los extraños acerca de la empresa), afectan la identificación de las personas. Desde el punto de vista de la marca interna y la marca del empleador hacia una audiencia externa, la marca corporativa podría ser más fuerte y más consistente (Sivertzen, et al., 2013). El EB destaca los aspectos diferenciadores de las ofertas de empleo o el entorno de la organización (Eger, et al., 2019; Sivertzen, et al.,2013). En general, una imagen positiva de la marca del empleador genera atractivo organizacional y el atractivo significa congruencia entre los valores y objetivos de la organización y los de los potenciales colaboradores (Eger, et al., 2019).

Si bien en los últimos años han proliferado estudios teóricos y empíricos respecto de la marca del empleador, es foco de esta investigación aportar con herramientas para la fortalecer la marca del empleador en zonas no metropolitanas, y en futuros colaboradores observando diferencias de género. Por otra parte, tal como lo afirman Babikova y Bucek (2019), es importante la replicación de estudios ya que "protege el campo académico de la aceptación acrítica de los resultados empíricos y es esencial especialmente para los campos emergentes, entre los cuales se considera la marca del empleador" (p.7).

\section{Formulación de hipótesis}

A partir de la revisión de las referencias, se formularon las hipótesis presentadas en la Tabla 1 para este estudio.

Tabla 1: Hipótesis para este estudio.

\begin{tabular}{|l|l|}
\hline Hipótesis & Detalle \\
\hline $\mathrm{H} 1$ & $\begin{array}{l}\text { Los estudiantes y egresados de las escuelas de negocio de Talca, al momento de } \\
\text { elegir su futuro lugar de trabajo, privilegian otros beneficios por sobre los económicos. }\end{array}$ \\
\hline $\mathrm{H} 2$ & $\begin{array}{l}\text { Entre los estudiantes y egresados de las escuelas de negocio de Talca, no existen } \\
\text { diferencias por género al momento elegir el futuro lugar de trabajo. }\end{array}$ \\
\hline $\mathrm{H} 3$ & $\begin{array}{l}\text { Los estudiantes que aún no egresan, le dan mayor valor a un trabajo emocionante, } \\
\text { novedoso y creativo, por sobre otras variables. }\end{array}$ \\
\hline
\end{tabular}

\section{METODOLOGÍA}

Esta investigación tiene un enfoque cuantitativo no experimental, cuya finalidad es observar situaciones ya existentes no provocadas intencionalmente por el investigador, y que se utiliza para conocer las dimensiones de la marca del empleador. Si bien es un diseño de investigación de tipo descriptivo, es un estudio con ribetes de exploración. Se utiliza la recolección y el análisis de datos para contestar la pregunta de investigación. A continuación, se exponen en el apartado de metodología, el instrumento de evaluación, la muestra y el trabajo de campo.

\section{Instrumento de evaluación}

Para este estudio se utilizó la herramienta EmpAt (Escala de Atractivo del Empleador) de Berthon, et al., (2005), desarrollada para medir la estrategia de la marca del empleador (EB). Esta escala se utilizó porque identifica, operacionaliza y mide los factores más relevantes del EB, desde la perspectiva de los potenciales 
colaboradores, utilizando una escala de medición creada en base a dichos factores. Entre las ventajas de trabajar con esta herramienta es que ha sido ampliamente utilizada en investigaciones sobre esta temática, lo cual permite generar comparaciones con otros estudios realizados por ejemplo en contextos culturales diferentes (Eger, et al., 2019). En relación a las dimensiones del EB, Berthon, et al., (2005), hicieron una revisión literaria e implementaron grupos focales, definiéndose 32 ítems de atracción laboral, que luego fueron aplicados a una muestra por conveniencia de 683 estudiantes. Posteriormente, se sometieron los resultados a una prueba de purificación en base al Alfa de Cronbach, depurándose en 25 elementos como resultado del análisis factorial, con un nivel de confiabilidad estadística de 0,96. Los elementos se agruparon en las siguientes cinco variables: (1) El valor del interés, que mide la atracción en base a un ambiente de trabajo emocionante, prácticas de trabajos novedosa y el valor de la creatividad; (2) El valor social, que mide la atracción en base a un ambiente divertido, feliz, de buenas relaciones con los pares; (3) El valor económico, que mide la atracción respecto a salarios, compensación y seguridad laboral; (4) El valor de desarrollo, que mide la atracción según el reconocimiento, la confianza y autoestima junto con las oportunidades de desarrollo y ascenso laborales; y (5) Valor de aplicación, que mide la atracción con relación a la oportunidad que da el empleador, para que el trabajador puede aplicar sus conocimientos y capacidades, tanto como para satisfacción del cliente como para complementar conocimientos de los compañeros de trabajo (Berthon, et al., 2005; Liu, 2018; Sivertzen, et al., 2013). Para la medición de los ítems señalados, se usó una escala Likert con 7 niveles, partiendo desde 1 (nada importante), hasta 7 (máxima importancia). Cada ítem fue traducido desde el idioma inglés al español. El estudio se complementó a su vez, con información sociodemográfica de los encuestados. Finalmente, se incorporaron otras dos variables para medir la estrategia de EB, la primera extraída de la investigación de Gavilan y Avello (2011) sobre valor de la marca empleadora, y una segunda variable para medir el valor de la cultura, obtenida a partir del estudio "Denison Organizational Culture Survey" de Bonavia, et al., (2009). Estas dos variables se definen de la siguiente forma: (1) Valor de conocimiento de la marca empleadora, que mide los principales componentes internos y externos de las empresas, sobre los cuales los futuros colaboradores poseen mayor interés; y (2) Valor cultural, que mide la importancia que posee la cultura organizacional en los trabajadores, siendo los valores, comportamiento organizacional, estilo de dirección, liderazgo entre otros, parte de este componente.

\section{Muestra y Trabajo de campo}

Esta investigación se desarrolló en la ciudad de Talca, región del Maule, entre los meses de agosto y septiembre de 2018, encuestándose a estudiantes y titulados de las principales escuelas de negocio de la región, provenientes de la Universidad Católica del Maule, la Universidad de Talca y la Universidad Autónoma de Chile. Además, se estableció como requisito para la muestra, que las carreras tuviesen alumnos egresados al año 2017 o en proceso de titulación. De esta forma, se seleccionaron las profesiones de Ingeniería Comercial, Contador Público y Auditor, Ingeniería Informática Empresarial, Ingeniería Civil Industrial e Ingeniería en Control de Gestión.

Se realizó un muestreo no probabilístico por juicio. El instrumento de medición se aplicó a 298 individuos, pertenecientes a tres universidades de la región del Maule (Autónoma, De Talca y Católica del Maule) y que ingresaron entre los años 2014 y 2015 y que egresaron de alguna de ellas. Esta muestra sobre la que se trabajó son los individuos que se pudieron contactar y que tienen la información que la investigación requiere, en la unidad de muestra que en este caso son los departamentos o escuelas de las Universidades en estudio. Por otra parte, del total de la muestra, se seleccionó proporcionalmente, de acuerdo a la cantidad de sujetos de estudio para cada unidad de análisis, quedando el número de encuestados según universidad: 46 individuos pertenecientes a la Universidad Católica del Maule,152 a la Universidad de Talca y 100 a la Universidad Autónoma de Chile.

Lo anterior, en base a la proporción por alumnos y egresados a encuestar para realizar el análisis factorial. Para aplicar las encuestas se contactó a diferentes autoridades de las universidades, se ocuparon las bases de datos de recién egresados y de alumnos de quinto año, encuestándose vía online, en la plataforma de alumnos o exalumnos, según sea el caso. El trabajo de campo se prolongó por un periodo de tres meses.

\section{RESULTADOS}

La técnica de interpretación del estudio fue a través de un análisis factorial de los componentes principales con rotación Varimax, con el objetivo de reducir la cantidad de variables a menos factores que explicaran las dimensiones de la atracción y retención laboral. Los resultados de la clasificación de variables sociodemográficas determinaron que las edades de los encuestados estaban dentro de un rango mínimo de 21 años y un rango máximo de 40 años. Con una media de 23,9 años y una desviación estándar de 2,348 años. 
La muestra obtenida corresponde a 298 encuestados, los cuales pertenecen a las carreras del área de negocios impartidas en las tres principales universidades de la región del Maule, entre ellos a 164 participantes de Ingeniería Comercial donde predominan las mujeres, ya que conciernen al $51,2 \%$ de la muestra. Los estudiantes de pregrado corresponden a un $75 \%$ del total de encuestados, mientras que los egresados serian de $25 \%$. Finalmente, la concentración máxima de respondientes, corresponde a alumnos de pregrado que están cursando cuarto año de la carrera de Ingeniería Comercial en la Universidad de Talca, ver detalle en Tabla 2. Con relación a la matriz de correlaciones de las variables en estudio, esta arrojó un resultado de 0,144 lo que indica que el análisis es factible. Asimismo, la prueba de KMO arrojó (.776) por lo tanto como es mayor que 0.5 es aceptable para el análisis factorial. La prueba de Bartlett (.000) es menor que 0.05 con lo cual es adecuado el realizar el análisis factorial. Y finalmente un Alfa de Cronbach de 0,736 lo que quiere decir que la escala es fiable, ya que los ítems poseen una muy buena correlación entre sí (AdasmeBerríos, et al., 2013)

Tabla 2: Estadísticos descriptivos variable sociodemográficas

\begin{tabular}{|l|l|l|l|}
\hline Variable & Valores & Recuento & Porcentaje \\
\hline \multirow{5}{*}{ Sexo } & Hombre & 155 & $52 \%$ \\
\cline { 2 - 4 } & Mujer & 143 & $48 \%$ \\
\cline { 2 - 4 } & Total & 298 & $100 \%$ \\
\hline \multirow{5}{*}{ Universidad } & Universidad Católica del Maule & 46 & $15 \%$ \\
\cline { 2 - 4 } & Universidad de Talca & 152 & $51 \%$ \\
\cline { 2 - 4 } & Universidad Autónoma & 100 & $34 \%$ \\
\cline { 2 - 4 } & Total & 298 & $100 \%$ \\
\hline & Ingeniería Comercial & 164 & $55 \%$ \\
\cline { 2 - 4 } & Ingeniería en Informática Empresarial & 27 & $9 \%$ \\
\cline { 2 - 4 } & Auditoría & 18 & $6 \%$ \\
\cline { 2 - 4 } & Ingeniería Civil Industrial & 27 & $9 \%$ \\
\cline { 2 - 4 } & Auditoría e Ingeniería en Control de Gestión & 56 & $19 \%$ \\
\cline { 2 - 4 } & Contador Público y Auditor & 6 & $2 \%$ \\
\cline { 2 - 4 } & Total & 298 & $100 \%$ \\
\hline \multirow{3}{*}{ Situación } & Matrícula 2014 & 108 & $36 \%$ \\
\cline { 2 - 4 } & Matrícula 2015 & 116 & $39 \%$ \\
\cline { 2 - 4 } & Egresados & 74 & $100 \%$ \\
\cline { 2 - 4 } & Total & 298 & \\
\hline
\end{tabular}

A continuación, en la Tabla 3, se presenta la varianza explicada, el método de extracción: Análisis de Componentes principales. En este análisis se consideran los factores que expliquen la mayor cantidad de varianza, con ello se determina que 4 factores explican un $47,5 \%$ de la varianza de los datos obtenidos, así se redujeron los 42 componentes de la encuesta para explicar la totalidad de las dimensiones y los datos entregados.

Tabla 3: Varianza Explicada

\begin{tabular}{|c|c|c|c|c|c|c|c|c|c|}
\hline \multicolumn{10}{|c|}{ Varianza total explicada } \\
\hline \multirow[t]{2}{*}{ Componente } & \multicolumn{3}{|c|}{ Auto valores iniciales } & \multicolumn{3}{|c|}{$\begin{array}{l}\text { Sumas de las saturaciones al } \\
\text { cuadrado de la extracción }\end{array}$} & \multicolumn{3}{|c|}{$\begin{array}{l}\text { Suma de las saturaciones al } \\
\text { cuadrado de la rotación }\end{array}$} \\
\hline & Total & $\begin{array}{l}\% \text { de la } \\
\text { varianza }\end{array}$ & $\begin{array}{l}\% \\
\text { acumulado }\end{array}$ & Total & $\begin{array}{l}\% \text { de la } \\
\text { varianza }\end{array}$ & $\begin{array}{l}\% \\
\text { acumulado }\end{array}$ & Total & $\begin{array}{l}\% \text { de la } \\
\text { varianza }\end{array}$ & $\begin{array}{l}\% \\
\text { acumulado }\end{array}$ \\
\hline 1 & 3,233 & 21,556 & 21,556 & 3,233 & 21,556 & 21,556 & 1,912 & 12,748 & 12,748 \\
\hline 2 & 1,383 & 9,223 & 30,778 & 1,383 & 9,223 & 30,778 & 1,879 & 12,530 & 25,278 \\
\hline 3 & 1,331 & 8,872 & 39,650 & 1,331 & 8,872 & 39,650 & 1,780 & 11,864 & 37,142 \\
\hline 4 & 1,183 & 7,885 & 47,535 & 1,183 & 7,885 & 47,535 & 1,559 & 10,394 & 47,535 \\
\hline
\end{tabular}

La matriz de componentes rotados presentada a continuación, en la Tabla 4, recoge de mayor a menor la carga de cada ítem en cada uno de los factores, comparando las saturaciones relativas de cada variable, en las cuatro dimensiones determinadas, de esta manera se aprecia cómo están constituidas cada una de ellas. El método de extracción utilizado es el análisis de componentes principales, y el método de rotación, la normalización Varimax con Kaiser. 
Tabla 4: Matriz de componentes rotados

\begin{tabular}{|c|c|c|c|c|}
\hline \multirow[b]{2}{*}{ Variables } & \multicolumn{4}{|c|}{ Factores } \\
\hline & $\begin{array}{l}\text { Valor del } \\
\text { desarrollo }\end{array}$ & $\begin{array}{c}\text { Valor } \\
\text { económico }\end{array}$ & Valor social & Valor del interés \\
\hline $\begin{array}{l}\text { 29. Conocer las marcas comerciales de la } \\
\text { organización }\end{array}$ &, 723 &, 109 & 014 & ,213 \\
\hline $\begin{array}{l}\text { 31. Conocer los países en los que opera la } \\
\text { organización }\end{array}$ & ,692 & 094 & ,047 &,- 087 \\
\hline $\begin{array}{l}\text { 28. Conocer el sueldo que voy a percibir en la } \\
\text { organización }\end{array}$ & 671 & ,049 & ,215 & ,021 \\
\hline $\begin{array}{l}\text { 30. Conocer los principales competidores de la } \\
\text { organización }\end{array}$ & ,492 & ,166 & ,004 & ,158 \\
\hline $\begin{array}{l}\text { 24. Que la organización ofrezca un salario } \\
\text { superior al promedio }\end{array}$ &,- 013 & ,702 & ,083 & ,092 \\
\hline $\begin{array}{l}\text { 21. Que la organización ofrezca estabilidad } \\
\text { laboral }\end{array}$ & ,136 & ,668 &,- 002 & ,136 \\
\hline $\begin{array}{l}\text { 15. Que la organización entregue } \\
\text { oportunidades de promoción dentro de ella }\end{array}$ & ,248 & ,617 & ,289 &,- 171 \\
\hline $\begin{array}{l}\text { 25. Que la organización ofrezca un atractivo } \\
\text { paquete de beneficios adicionales al } \\
\text { sueldo }\end{array}$ & ,233 & ,517 & , 146 & ,154 \\
\hline $\begin{array}{l}\text { 7. Tener una buena relación con sus } \\
\text { superiores }\end{array}$ &,- 073 & ,288 & ,673 &,- 100 \\
\hline 2. Un ambiente de trabajo agradable & 071 &,- 183 & ,646 & ,254 \\
\hline $\begin{array}{l}\text { 9. Contar con compañeros que entreguen } \\
\text { apoyo y aliento }\end{array}$ & ,220 &, 150 & 611 & ,013 \\
\hline 8. Tener una buena relación con sus colegas & ,069 & ,168 &, 565 & ,242 \\
\hline $\begin{array}{l}\text { 11. Contar con jefes que implementen } \\
\text { prácticas de trabajo innovadoras y que } \\
\text { tengan pensamiento vanguardista }\end{array}$ & ,099 & ,078 &, 112 & 675 \\
\hline 10. Trabajar en un ambiente dinámico & ,020 & ,255 & ,049 & ,657 \\
\hline $\begin{array}{l}\text { 12. Que la organización valore la creatividad } \\
\text { de los colaboradores y haga uso de ella }\end{array}$ &, 102 &,-063 & ,202 & ,616 \\
\hline KMO & & & & ,776 \\
\hline Bartlett test & & & &, 000 \\
\hline
\end{tabular}

El análisis de confiabilidad se determinó a través del Alfa de Cronbach, cuyo valor fue de 0,736, $\alpha>0.7$ (Hair et al., 1999). En la Figura 1, Gráfico de sedimentación se aprecia que los valores mayores que 1 corresponden a 4 , o sea 4 factores explicarán toda la información obtenida y con ello resumirán al resto de los componentes.

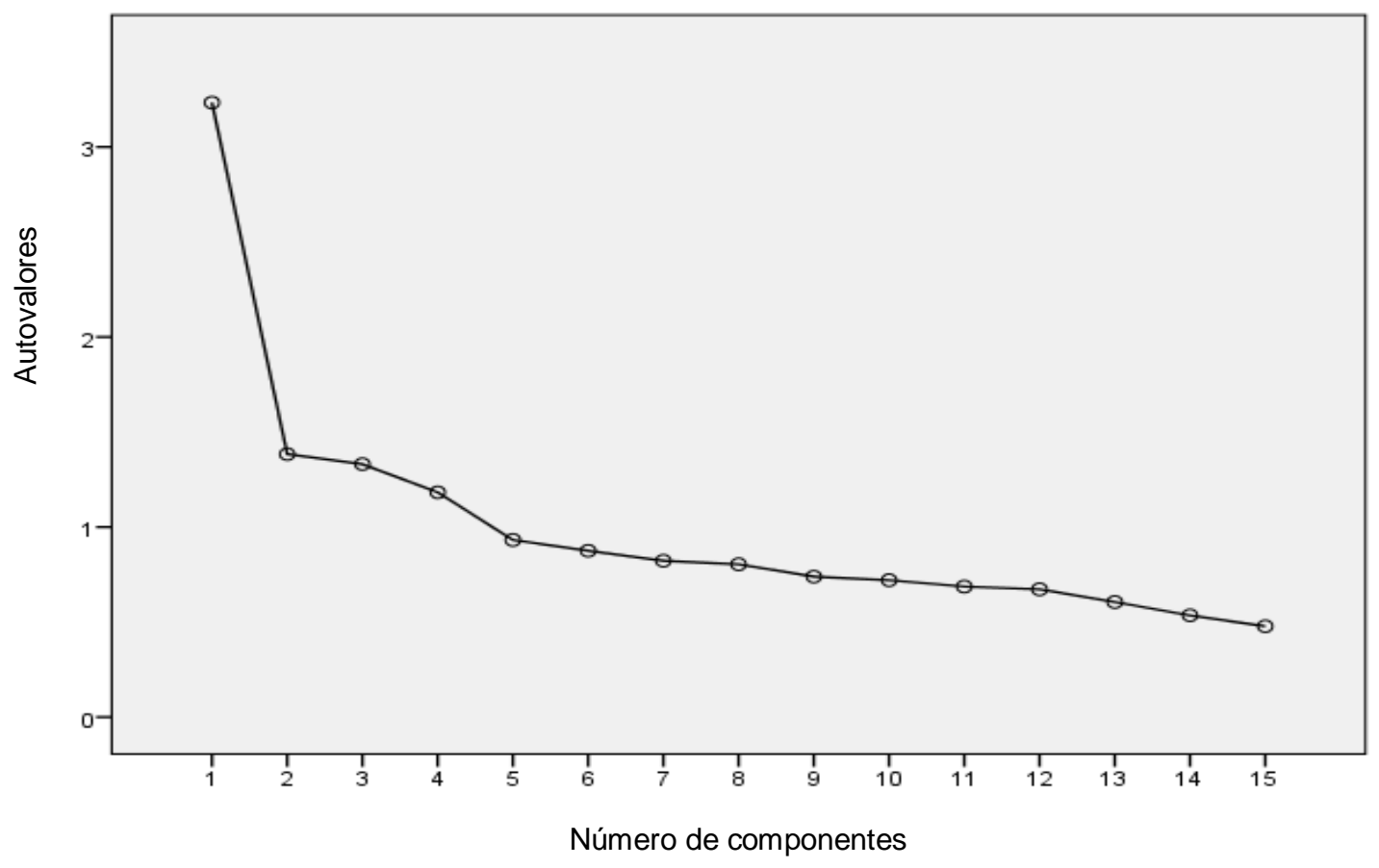

Fig. 1: Gráfico de sedimentación 


\section{Factores determinados globales}

Según la matriz de componentes rotados resultante de todos los encuestados, se identificaron las variables y se agruparon en factores que poseen una carga mayor a 0,5, extrayéndose las que no poseían esta condición. En otras palabras, se realizó una depuración de componentes los cuales no llegaban al rango que se estableció como aceptable.

Tabla 5: Factores y sus principales variables

\begin{tabular}{|l|l|c|}
\hline Factores & \multicolumn{1}{|c|}{ Variables } & Peso \\
\hline \multirow{4}{*}{ Valor de } & a) Conocer las marcas comerciales de la organización & $(, 723)$ \\
desarrollo & b) Conocer los países en los que opera la organización & $(, 0692)$ \\
& c) Conocer el sueldo que voy a percibir en la organización & $(, 671)$ \\
& d) Conocer los principales competidores de la organización & $(, 492)$ \\
\hline \multirow{5}{*}{ Valor } & a) Salario ofrecido por la organización superior al promedio & $(, 702)$ \\
económico & b) Estabilidad laboral ofrecida por la organización & $(, 668)$ \\
& c) Oportunidades de promoción interna entregadas por la organización & $(, 617)$ \\
& d) Atractivo paquete de beneficios adicionales al sueldo entregado por la & $(, 517)$ \\
\hline \multirow{5}{*}{ Valor social } & organización. & $(, 673)$ \\
& a) Tener una buena relación con sus superiores & $(, 643)$ \\
\hline \multirow{5}{*}{$\begin{array}{l}\text { Valor del } \\
\text { interés }\end{array}$} & b) Ambiente de trabajo agradable, & $(, 611)$ \\
& d) Tentar con compañeros que entreguen apoyo y aliento & $(, 565)$ \\
\hline & a) Contar con jefes que implementen prácticas de trabajo innovadoras y que & $(, 675)$ \\
& tengan pensamiento vanguardista. & $(, 657)$ \\
& b) Trabajar en un ambiente dinámico. & $(, 616)$ \\
\hline
\end{tabular}

Se realizó un análisis de las variables que tributarían al factor género y egreso, tal como se presenta a continuación.

Tabla 6: Factor por género y sus principales componentes

\begin{tabular}{|c|c|c|}
\hline $\begin{array}{l}\text { Género } \\
\text { Masculino } \\
\mathrm{KMO}=0,8 \\
\text { Alfa de } \\
\text { Cronbach= } \\
0,801\end{array}$ & a) & $\begin{array}{l}\text { Predominancia al valor de interés, ellos se sienten atraídos por la novedad, ya sea en prácticas } \\
\text { laborales y/o productos y servicios. Del mismo modo poseen atracción por el valor cultural y los } \\
\text { valores de la marca empleadora. } \\
\text { Enfoque al valor de aplicación, este se enfoca en obtener experiencia laboral, valoración de la } \\
\text { organización a los colaboradores, que desarrollen sentido de pertenencia y con ello se sientan más } \\
\text { seguros de sí mismos. } \\
\text { Valor de desarrollo, variables que componen el valor de la marca empleadora y sus factores } \\
\text { externos, es decir los hombres poseen gran interés en dónde se desempeña la organización, sus } \\
\text { marcas comerciales más atractivas y un factor interno de conocer el sueldo que percibirá el } \\
\text { colaborador. }\end{array}$ \\
\hline $\begin{array}{l}\text { Género } \\
\text { Femenino } \\
\text { KMO=0,705 } \\
\text { Alpha de } \\
\text { Cronbach }= \\
0,733 \text {, }\end{array}$ & $\begin{array}{l}\text { a) } \\
\text { b) }\end{array}$ & $\begin{array}{l}\text { Predomina el valor de desarrollo, la relación con el conocimiento de la marca empleadora, donde } \\
\text { sus principales intereses son los valores externos e internos. } \\
\text { El segundo factor corresponde al valor social, el cual se preocupa por el ambiente de trabajo, la } \\
\text { estabilidad, reconocimiento y apreciación por parte de las jefaturas a las colaboradoras y tener una } \\
\text { buena relación con sus pares. } \\
\text { La tercera dimensión es el valor de interés, es decir se siente atraída por el empleador gracias a } \\
\text { pertenecer y trabajar en un ambiente dinámico. }\end{array}$ \\
\hline
\end{tabular}

En relación a los egresados, no se pudo determinar los factores de atracción ya que subdividiendo en esta característica la cantidad de encuestados disminuye en gran parte la muestra, haciendo el análisis factorial poco fiable.

\section{DISCUSIÓN}

Este estudio permite identificar las dimensiones más valoradas de la estrategia del EB, para los futuros profesionales del área de negocios de la región del Maule, pretendiendo convertirse en información útil para poder realizar una eficiente gestión de contratación y atracción de recursos humanos. 
Sobre la validez de los resultados podemos concluir que la primera hipótesis establecida no es aceptada, puesto que como se señala anteriormente la variable más importante para los egresados es el conocimiento de la marca por sobre los beneficios económicos, es decir, en este caso el factor de desarrollo prima por sobre los otros factores, indicando que para los jóvenes es atractivo ingresar a trabajar a una organización que posea una marca reconocida, ya que esto les permitirá optar a oportunidades de desarrollo, reconocimiento, entre otros. Al comparar estos resultados con otras investigaciones, es posible notar que hay concordancias, en relación a que la reputación de la empresa contribuye positivamente a las intenciones de solicitud de empleo del grupo objetivo, (Liu, 2018; Sivertzen, et al., 2013) coincidiendo en el factor más relevante. Asimismo, en el estudio Kucherov, et al., (2019), si bien el factor económico no es el más relevante, ya que en sus conclusiones dejan de manifiesto que el más importante para los jóvenes checos es el valor social, relevan que para los millenials, es muy significativo el perfil corporativo general del empleador, indicando que están dispuestos incluso a recibir un salario menor, por trabajar en una empresa que tenga el atributo de conocimiento de marca en el mercado. En la misma línea están los hallazgos de Alniaçik, et al. (2012), quienes concluyen que el valor social es el factor más atractivo, seguido por el factor de conocimiento de la empresa. Como se puede observar, ambos estudios difieren de los hallazgos de la presente investigación, donde el factor de desarrollo, se presenta como la dimensión de mayor relevancia. Por otra parte, hay diferencias más profundas con otros autores, en el caso del estudio de Glufke (2015), se indicó que la dimensión más importante para la generación de millennials, era el valor económico, factor que estaría compuesto por un salario superior a la media, con compensaciones atractivas y ascensos laborales.

Este resultado es importante, ya que orienta a quienes tienen en sus manos los procesos de reclutamiento y selección de personas, para realizar un análisis más profundo de los factores que más relevan e influyen en los potenciales colaboradores, y de esta manera puedan generar estrategias para atraer los mejores talentos. Además, la relevancia de que esta variable sea reconocida como la más importante, radica en que, facilita la alineación del futuro colaborador con la misión, visión y objetivos de la organización.

En relación a la segunda hipótesis, también se rechaza, ya que es posible observar una diferencia de prioridades entre los diferentes sexos, este estudio permite concluir que entre los hombres predomina el valor de interés, es decir los hombres poseen gran interés en donde se desempeña la organización, ya que privilegian que exista innovación y ambientes dinámicos. Además, relevan variables de otras dimensiones, tales como, marcas comerciales atractivas y un factor interno de conocer el sueldo que percibirá el colaborador. Por su parte, el género femenino se inclinó por el factor de desarrollo a través del conocimiento de la marca empleadora, luego, el factor social y el factor de interés. En general, se podría apelar a un comportamiento cultural, donde el rol masculino se asocia más al riesgo y la innovación que el femenino. En el estudio realizado por Alniaçik et al., (2012), no se encontró ninguna diferencia significativa entre los encuestados masculinos y femeninos. Sin embargo, nuevamente hay coincidencias con la investigación de Kucherov, et al., (2019), quienes encontraron diferencias entre ambos sexos, aunque las dimensiones prioritarias no coinciden, otorgando las mujeres mayor importancia al factor social. En general las organizaciones requieren tener equipos diversos para lograr mejorar su desempeño, en este caso la diversidad viene dada por la diferencia en las prioridades de valorización de la marca del empleador según el género.

Por otra parte, los estudiantes se mostraron más dispuestos a trabajar en organizaciones creativas y dinámicas, donde el clima laboral sea grato, a conocer las políticas de horarios, y la importancia de la responsabilidad social sin abarcar el factor económico. De esta forma es posible aceptar la tercera hipótesis, y concluir que los futuros colaboradores le dan más importancia a la dimensión de interés que a los beneficios económicos. Este resultado es concordante con lo expuesto por Kucherov, et al., (2019), que indican que los jóvenes son sensibles al perfil corporativo, y que prefieren organizaciones que operan en entornos cambiantes y mercados dinámicos. Claramente, este resultado viene a constatar las características del perfil de personas a las que apuntó la investigación, jóvenes que valoran un estilo de vida independiente, autosuficiente, que les gusta trabajar en equipos y en organizaciones que vean más allá de la rentabilidad económica. Conocer este resultado es clave para tener las claves para orientar las estrategias de reclutamiento de talento, así como de mantención del mismo.

En resumen, la variable que más valoraron los jóvenes fue la del conocimiento de marca, es decir consideran importante que la empresa en la cual ellos van a trabajar sea conocida y tenga prestigio. Por otra parte, este estudio evidencia la diferencia que existe entre los jóvenes, en relación a la valoración de los factores según género, donde las mujeres valoran más fuertemente a las organizaciones que dan reconocimiento, autoestima y confianza; mientras que, los hombres dan más importancia al ambiente de trabajo innovador, dinámico, competitivo, donde puedan usar su creatividad. Finalmente, los estudiantes que aún no egresan de la universidad, valoran más los ambientes de trabajo innovadores, creativos, responsables socialmente y con ambientes de trabajo saludables, y le dan mayor importancia por sobre el valor económico. 
Con respecto a las limitaciones, éstas se presentan en base a la muestra, ya que solo cuenta con estudiantes y egresados de las carreras de negocios en un rango de edad relativamente corto, por lo que este estudio no captura la información en relación a la atracción de personas de otros rangos etarios o de otras áreas laborales diferentes al área de negocios, esto también puede discrepar con el instrumento utilizado, ya que como indica el autor el hecho de que la herramienta haya sido creada, probada y aplicada principalmente en carreras del área comercial quizás no sea representativa y efectiva en otras áreas laborales. Respecto al ámbito de este estudio, sería deseable realizar la investigación con el mismo perfil de candidatos, pero con enfoque sólo en los egresados ya que no se pudo determinar los factores de atracción debido que subdividiendo la muestra en esta característica la cantidad de encuestados disminuye en gran parte, haciendo el análisis factorial poco fiable.

\section{CONCLUSIONES}

La marca del empleador se ha convertido en una estrategia que cada vez implementan más organizaciones, de manera de poder aprovechar sus ventajas. De los resultados, análisis y discusión, presentados previamente, se obtienen las siguientes conclusiones: 1) El hecho de que los jóvenes valoren el conocimiento de marca, al ser un fuerte símbolo de identidad organizacional facilita la alineación con las directrices estratégicas, al momento de incorporar o retener al talento en la empresa. 2) Las estrategias que deben proponer las organizaciones para retener y atraer talento deben considerar la diferencia de valoración de factores por género, donde las mujeres esperan potenciar una carrera laboral en la organización al visualizar su desarrollo en futuros empleos y los hombres buscan entornos laborales más desafiantes, esta diferencia se podría relacionar con un patrón cultural asociado a la crianza y el entorno social. 3) Las organizaciones deben considerar las necesidades de las nuevas generaciones si esperan atraer y retener al mejor talento. Esto es muy relevante, principalmente al estar insertos en un entorno altamente competitivo y orientado a la economía digital. Finalmente, 4) esta investigación aporta al campo científico nueva evidencia en relación a la valoración de factores por sexo, además de la replicabilidad en Chile.

\section{REFERENCIAS}

Adasme-Berríos, C.E., Jara-Rojas, R.A., Ramos-Cabello,B.N., Rodríguez, M.A., y Mora, M.G., Consumer Responses to Agricultural Produce Advertising in the O'Higgins Region, Chile. Cien. Inv. Agr., 40(1), 31-41 (2013)

Albert-Gómez, M.J., García-Pérez, M.M, y Pérez-Molina, C.M. Competencias, Formación y Empleo. Análisis de Necesidades en un Programa de Master en Ingeniería, http://dx.doi.org/10.4067/S0718-50062017000200006 , Formación Universitaria, 10(2), 43-56 (2017).

Alniaçik, E., Alniaçik, Ü. Y Erdogmus, N., How do the Dimensions of Corporate Reputation Affect Employment Intentions? Corp Reputation Review, 15, 3-19 (2012)

Ambler, T. y Barrow, S., The Employer Brand, The Journal of Brand Management, 4(3), 185-206 (1996)

Babikova, K. y Bucek, J., A Model Replication with an Extension of Students' Perception of Prospective Employer Attractiveness, https://doi.org/10.7441/joc.2019.02.01 Journal of Competitiveness, 11(2), 5-21 (2019)

Backhaus, K. y Tikoo, S., Conceptualizing and Researching Employer Branding, https://doi.org/10.1108/13620430410550754, Career Development International, 9(5), 501-517 (2004)

Backhaus, K., Employer Branding Revisited, https://doi.org/10.1080/15416518.2016.1245128, Organization Management Journal, 13(4), 193-201 (2016)

Berthon, P., Ewing, M. y Hash, L., Captivating Company: Dimensions of Attractiveness in Employer Branding, https://doi.org/10.1080/02650487.2005.11072912 International Journal of Advertising, 4(2), 151-153 (2005)

Bonavia, T., Prado, V.J., y Barberá, T.D., Spanish adaptation and factor structure of the Denison Organizational Culture Survey, Psicothema, 21(4), 633-638 (2009)

Brymer, R.A., Molloy, J.C., y Gilbert, B.A., Human Capital Pipelines Competitive Implications of Repeated Interorganizational Hiring, https://doi.org/10.1177/0149206313516797 Journal of Management, 40(2), 483-508 (2014)

Bussin, M. y Mouton, H., Effectiveness of Employer Branding on Staff Retention and Compensation Expectations, https://doi.org/10.4102/sajems.v22i1.2412 South African Journal of Economic and Management Sciences, 22(1), 1-8 (2019)

Deepa R. y Baral R., A Comprehensive Framework for Implementing an Effective Employer Brand Strategy, https://doi.org/10.1177/0972150917693152, Global Business Review, 18(3), 83-85 (2017)

Deloitte Chile, The 2018 Deloitte Millennial Survey. Deloitte. (2018).

Eger, L., Micik, M., Gangur, M., y Řehor, P., Employer Branding: Exploring Attractiveness Dimensions in a Multicultural Context, https://doi.org/10.3846/tede.2019.9387, Technological and Economic Development of Economy, 25(3), 519-541 (2019) 
Glufke, G., Braga, B.M., Employer Attractiveness from a Generational Perspective: Implications for Employer Branding, https://doi.org/10.5700/rausp1226, Revista de Administração, 51, 103-116 (2015)

Hair J. R., y Tatham, W., Análisis Multivariante. 5th ed., Prentice Hall Iberia., Madrid, España (1999)

INE, Boletín estadístico, Región del Maule, Instituto Nacional de Estadísticas Chile, Santiago, Chile (2018)

Kucherov, D.G., Zamulin, A.L., y Tsybova, V.S. How Young Professionals Choose Companies: Employer Brand and Salary Expectations, https://doi.org/10.21638/spbu18.2019.102, Russian Management Journal, 17(1), 29-46 (2019)

Liu, Y., Employer Attractiveness to Chinese Potential Employees, http://dx.doi.org/10.26417/ejis.v4i2.p147-155, European Journal of Interdisciplinary Studies, 4(2), 147-155 (2018).

Love, L.F. y Singh. P., Workplace Branding: Leveraging Human Resources Management Practices for Competitive Advantage through "Best Employer" Surveys, https://doi.org/10.1007/s10869-011-9226-5, Journal of Business and Psychology, 26(2), 175-181 (2011)

Maxwell, R. y Knox, S.D., Motivating Employees to "Live the Brand": a Comparative Case Study of Employer Brand Attractiveness within the Firm, https://doi.org/10.1362/026725709X479282, Journal of Marketing Management, 25(9-10), 893-907 (2009)

Mičík, M., y Mičudová, K., Employer Brand Building: Using Social Media and Career Websites to Attract Generation Y, https://doi.org/10.14254/2071-789X.2018/11-3/11, Economics and Sociology, 11(3), 171-189 (2018).

Miles, S.J. y Mangold, G. W., A Conceptualization of the Employee Branding Process. https://doi.org/10.1300/J366v03n02_05 Journal of Relationship Marketing, 3(2-3), 65-87 (2004)

Ramoni, J. y Orlandoni, G., Análisis de la Estructura del Mercado Laboral en Colombia: un Estudio por Género mediante Correspondencias múltiples, https://doi.org/10.1016/j.cesjef.2016.02.002 Cuadernos de Economía, 40(113), 100-114 (2017)

Sivertzen, A.M., Nilsen, E.R. y Olafsen, A.H., Employer Branding: Employer Attractiveness and the Use of Social Media, https://doi.org/10.1108/JPBM-09-2013-0393 Journal of Product \& Brand Management, 22(7), 473-483 (2013)

Veas-González, I.A., Sanchez-Ortiz, A.D., Perez-Cabañero, C., Determinantes del Marketing Boca a Boca en el Contexto Universitario: El Rol de la Satisfacción y la Lealtad de los Estudiantes, http://dx.doi.org/10.4067/S071850062019000100045 , Formación Universitaria,12(1), 45-54 (2019) 
